\title{
An unusual occurrence of solitary extramedullary plasmacytoma in the conjunctiva
}

\author{
JINGJING JIANG ${ }^{1}$, YONG LIU ${ }^{1}$, FULIN WANG ${ }^{2}$ and YIFEI HUANG ${ }^{1}$ \\ Departments of ${ }^{1}$ Ophthalmology and ${ }^{2}$ Pathology, \\ Chinese PLA General Hospital, Beijing, P.R. China
}

Received February 3, 2012; Accepted May 9, 2012

DOI: $10.3892 / \mathrm{ol} .2012 .726$

\begin{abstract}
The occurrence of a solitary plasmacytoma in the conjunctiva is unusual. This relatively uncommon neoplastic disorder occurs more frequently in other organs and is derived from B-lymphocytes. In the present study the case of a 17-yearold female, who had a lesion of the lower fornix conjunctiva in the left eye, is presented. The lesion was resected and the patient underwent amniotic membrane transplantation. Pathological examination of the specimen showed a plasmacytoma. Bone marrow aspirate showed that the cellularity and number of plasma cells were normal, and no myeloma cells were observed. A complete skeletal survey failed to show bony masses or osteolytic events. Following surgery, the patient was provided with a course of radiotherapy. During the follow-up period of 12 months the lesion did not recur.
\end{abstract}

\section{Introduction}

Solitary extramedullary plasmacytoma is a relatively rare neoplastic disorder arising from B-lymphocytes. Although solitary extramedullary plasmacytoma may occur in numerous organs, including the central nervous system, urinary bladder, thyroid, breast, testes, parotid glands and lymph nodes (1), to the best of our knowledge, the occurrence of plasmacytoma in the conjunctiva is unusual. In the present study, we present a case report of plasmacytoma confined to the conjunctiva of a 17-year-old female.

Correspondence to: Professor Yifei Huang, Department of Ophthalmology, Chinese PLA General Hospital, 28 Fuxing Road, Haidian District, Beijing 100853, P.R. China

E-mail: huangyf301@gmail.com

Key words: plasmacytoma, conjunctiva

\section{Case report}

Patient examination. A 17-year-old Chinese female was admitted to the Chinese PLA General Hospital with a progressively increasing painless swelling in the lower fornix conjunctiva of the left eye, which was first noticed two months prior to presentation. There was no history of ocular infection or trauma in the affected eye and the family history of the patient revealed no ocular problems. Upon examination, the visual acuity of the patient was 20/20 oculi uterque (OU). Located on the lower fornix conjunctiva of the left eye, without congestion of palpebral or bulbar conjunctiva, was a mobile, elastic, pink mass approximately $23 \times 13 \mathrm{~mm}$ in size and its surface was smooth (Fig. 1). The remaining ophthalmic examination was unremarkable.

Immunohistochemistry. A surgical excision of the lesion with amniotic membrane transplantation was performed. Under microscopic examination, the specimen revealed sheets of plasma cells with a high proliferative index, which were surrounded by connective tissue. A number of the plasma cells had mitotic figures, which demonstrated that binucleated plasma cells were also observed (Fig. 2A). Immunocytochemical staining showed that CD138, IgG and $\kappa$ light chain were present and $\lambda$ light chain was not (Fig. 2B, $\mathrm{C}$ and D). Further investigation with bone marrow aspirate showed that the cellularity and number of plasma cells were normal, without any myeloma cells. A complete skeletal survey failed to show bony masses or osteolytic events. Serum protein electrophoresis was within normal limits and urine was negative for Bence Jones protein.

Treatment. Following surgery, the patient received a course of radiotherapy (a dose of 40 Gy in 10 administrations) and was invited for a checkup once a month. On follow-up (12 months) there was no sign of local recurrence or the development of multiple myeloma.

\section{Discussion}

Primary solitary extramedullary plasmacytoma is rare and it may be diagnosed on the condition of no evidence of solitary plasmacytoma of bone or multiple myeloma, including the 


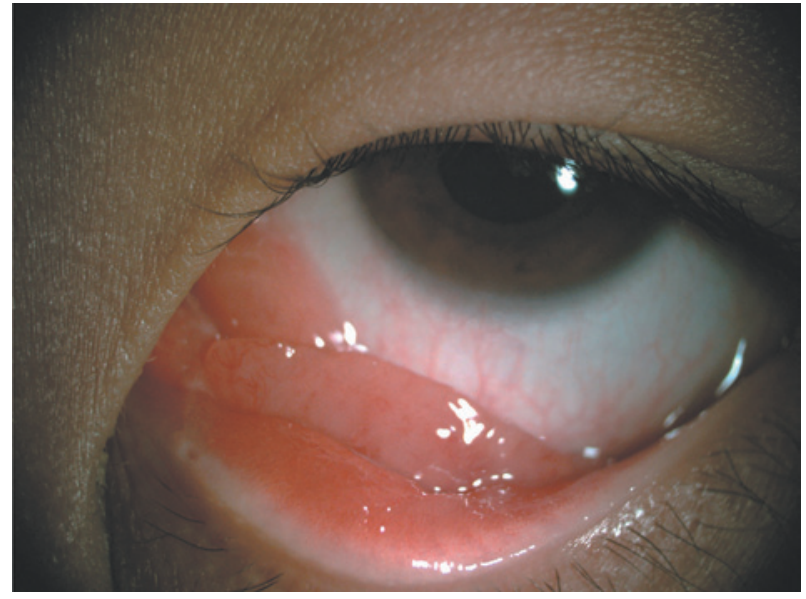

Figure 1. Left lower lid: a pink mass occupied the lower fornix of the eye.

bone marrow, without local tumour, and normal urine, serum electrophoresis and haemoglobin (2). The occurrence of plasmacytoma in the eye is infrequent. Olivieri et al (3) reported a case of eyelid plasmacytoma. Yumori et al (4) described a case of bulbar conjunctival plasmacytoma, which was not associated with clinical or laboratory signs of multiple myeloma. Certain cases report that plasmacytoma occurring in the eye is not true plasmacytoma, but instead is a granuloma due to chronic inflammation (5). Immunocytochemical staining may confirm diagnosis when the histopathological appearance is ambiguous. The origin of the plasmacytoma in the present case is unclear, but the plasmacytoma demonstrated a proliferation of CD138, CD79a and $\kappa$ light chain and the expression profiles were in accordance with plasmacytoma.

The prognosis for patients with plasmacytoma is favorable. Treatment includes surgery, radiotherapy and chemotherapy. The patient in this case was treated with surgery followed by radiotherapy, as bone marrow and blood examinations were normal and systemic evaluation did not reveal similar lesions elsewhere.
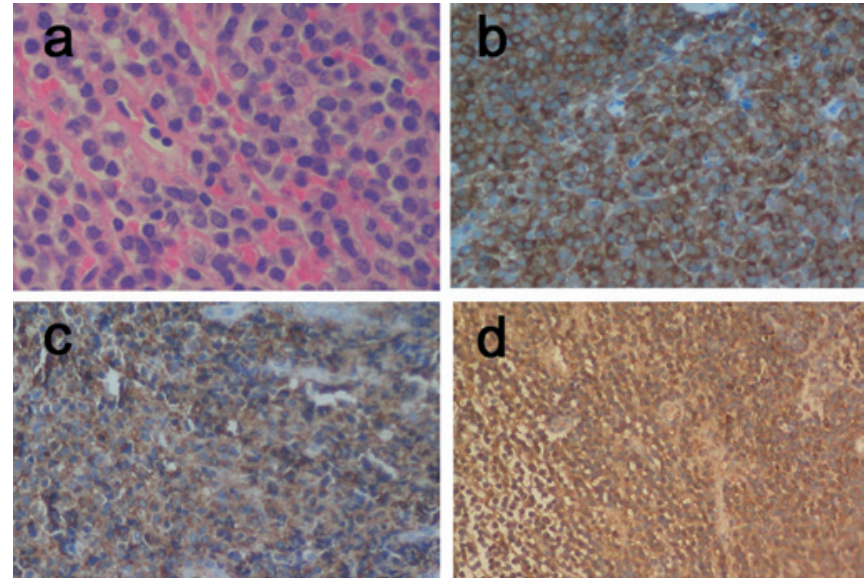

Figure 2. Histopathology. (A) Photomicrograph showing a pathological slide stained with hematoxylin and eosin. Pathological findings of plasma cells with a high proliferative index and mitotic figure are observed. Immunocytochemical staining for (B) CD79a, (C) CD138, (D) $\kappa$ light chain (magnification, x200).

In conclusion, we report a rare case of plasmacytoma originating in the fornix conjunctiva. No complications or tumor recurrence have been noted thus far. However, a longer follow up is required.

\section{References}

1. Dimopoulos MA, Kiamouris C and Moulopoulos LA: Solitary plasmacytoma of bone and extramedullary plasmacytoma. Hematol Oncol Clin North Am 13: 1249-1257, 1999.

2. Wax MK, Yun KJ and Omar RA: Extramedullary plasmacytomas of the head and neck. Otolaryngol Head Neck Surg 109: 877-885, 1993.

3. Olivieri L, Di Ianni M, Giansanti M, Falini B and Tabilio A: Primary eyelid plasmacytoma. Med Oncol 17: 74-75, 2000.

4. Yumori JW, Ilsen P and Bright DC: Conjunctival plasmacytoma. Optometry 81: 234-239, 2010.

5. Seddon JM, Corwin JM, Weiter JJ, Brisbane JU and Sutula FC: Solitary extramedullary plasmacytoma of the palpebral conjunctiva. Br J Ophthalmol 66: 450-454, 1982. 